\title{
Combined Upper Extremity and Gluteal Compartment Syndrome Following Illicit Drug Abuse: A Retrospective Case Series
}

This article was published in the following Dove Press journal: Orthopedic Research and Reviews

\section{Matthew W Konigsberg (D) John D Mueller Jordan A Lebovic R Kumar Kadiyala}

Department of Orthopedic Surgery, Columbia University Medical Center, New York, NY, USA
Correspondence: Matthew W Konigsberg Department of Orthopedic Surgery, Columbia University Medical Center, 622 W. I68th St. IIth Floor, New York, NY 10032, USA

Tel + I-2I 2-305-5976

Fax + I-2|2-305-6193

Email Mk3294@cumc.columbia.edu
Introduction: The purpose of this study was to describe four cases of patients who developed concomitant upper extremity and gluteal compartment syndrome in the context of substance abuse. In somnolent patients unable to provide a reliable physical exam, the healthcare provider must be aware of patients presenting with concomitant upper extremity and gluteal compartment syndrome.

Methods: Retrospective chart review identified cases of the combined upper extremity and gluteal compartment syndrome following illicit drug abuse at a single academic center during the years 2009-2019.

Results: During the 11-year period examined, a total of eight patients were diagnosed with compartment syndrome secondary to illicit drug use and prolonged immobilization. Four $(50 \%)$ patients presented with combined upper extremity and gluteal compartment syndrome. All of these patients underwent prompt surgical release of the affected compartments. All eventually returned to normal activities of daily living.

Discussion: Compartment syndrome is primarily a clinical diagnosis, with physical exam being extremely important. In patients presenting with somnolence secondary to illicit drug use, physical exam may not be reliable. It is critical to have a high clinical suspicion in this patient population, understanding that these patients may present with concomitant upper extremity and gluteal compartment syndrome.

Level of Evidence: Level IV, case series.

Keywords: compartment syndrome, fasciotomy, trauma, upper extremity, gluteal

\section{Introduction}

Acute compartment syndrome is a medical and surgical emergency that if left untreated can lead to significant morbidity and even mortality. ${ }^{1}$ Increased pressure within an enclosed fascial compartment can lead to ischemia and necrosis of the musculature and neurovascular structures within an affected compartment. The subsequent muscle breakdown can lead to rhabdomyolysis, renal failure, and death. This process is time sensitive, so early diagnosis and treatment is paramount. Even if a compartment syndrome is identified and treated in a timely manner, muscle and nerve damage may lead to permanent musculoskeletal impairments.

Illicit drug use has been associated with acute compartment syndrome as obtundation and immobility secondary to somnolence or loss of consciousness can lead to increased pressure within a specific compartment. This is important to emphasize in the setting of illicit drug use as these patients are often not able to 
fully participate in the physical exam or communicate when their pain and related symptoms arise. For any patient in which the clinician is suspicious of a compartment syndrome and the patient is unable to provide a reliable physical exam, measuring compartment pressures using an intra-compartmental pressure monitor may provide an alternative method of diagnosis. In this patient population, healthcare providers should have a high clinical suspicion for a compartment syndrome.

Cases of concomitant upper extremity and gluteal compartment syndrome are extremely rare and there is only one known case report described in the literature. ${ }^{2}$ In the United States, prolonged immobilization after alcohol or opioid abuse is a common etiology of compartment syndrome. ${ }^{3-7}$ In our cases, we have found that other illicit substances such as benzodiazepines, amphetamines and synthetic cannabinoids, which are less commonly described with compartment syndrome can also cause this presentation.

\section{Methods}

Medical records from a single academic center were reviewed from the years 2009-2019 to identify cases of compartment syndrome. Charts were reviewed for initial presentation including physical exam, vital signs on admission, and laboratory results in the emergency department. Also recorded for each patient were etiology of compartment syndrome (trauma, somnolence, other), specific compartments affected, surgical course, final followup exam and complications.

\section{Results}

At a large academic center from 2009 to 2019 there were eight cases of compartment syndrome secondary to substance abuse (out of a total of 64 cases of compartment syndrome). The average age was 43.75 years, and $50 \%$ of cases were male. Four of these cases $(50 \%)$ involved concomitant upper extremity and gluteal compartment syndrome. Each of these patients had the appropriate upper and lower extremity fasciotomies along with debridements.

\section{Case I}

A 31-year-old female with a history of polysubstance abuse (marijuana and cocaine) presented to the emergency department complaining of right upper and lower extremity numbness, pain and swelling for 1 day after using cocaine, marijuana, and benzodiazepines. She had fallen asleep on her right side for a number of hours and when she awoke, she had diffuse right-sided swelling of the arm, hand, leg and breast with significant pain, decreased sensation and functionality. She had difficulty ambulating. On arrival, vital signs were notable for tachycardia to 115 beats per minute. Her labs were notable for elevated liver and kidney function studies, as well as mild elevation of inflammatory markers, and creatine phosphokinase greater than 100,000 . On exam, right forearm compartments were found to be swollen, and the patient was noted to have a claw hand deformity and was unable to fire her intrinsic hand musculature. Her right lower extremity exam was notable for firm gluteal and leg compartments, though her thigh was noted to be soft and compressible. She had diminished sensation to light touch throughout her leg, and was unable to actively move her ankle or toes.

The patient was taken to the operating room emergently for fasciotomies of the right gluteal compartment, right leg compartments, right volar and dorsal forearm compartments, and right hand compartments, with subsequent wound vac placement. Throughout the hospitalization, she underwent multiple debridements and delayed primary closure of all fasciotomy incisions. The patient recovered all motor function of both her right upper and lower extremities, though had persistent paresthesias on the dorsum of the right foot at most recent follow-up 3 months postoperatively. She was able to resume all activities of daily living.

\section{Case 2}

A 40-year-old male with a history of polysubstance abuse (alcohol, opiates, benzodiazepines, tobacco, MDMA) presented to the emergency department complaining of pain and numbness in his left upper and lower extremity after an extended period of unconsciousness secondary to drug overdose. Vital signs upon arrival were within normal limits. Physical exam of the left upper extremity at that time was notable for pain, swelling, firm volar and dorsal forearm compartments, as well as no active motion of the wrist or fingers, and no sensation to light touch in the median, ulnar, and radial nerve distributions. The patient was also found to have firm gluteal, thigh and leg compartments (primarily anterior and lateral compartments) with complete loss of active ankle and toe motion and sensation to light touch in the deep peroneal nerve distribution. Urine toxicology was positive for THC, benzodiazepines and opiates. Labs were notable elevated kidney function markers as well as creatine phosphokinase greater 
than 100,000 . The patient emergently underwent fasciotomies of the left deltoid, pectoralis, volar and dorsal forearm, gluteus, thigh, lower leg with wound vac placement. The patient underwent serial debridements of the left upper and lower extremities, and eventually required application of a skin substitute bilayer. At most recent follow-up 5 months postoperatively, the patient had improving motor function with continued paresthesias in both the left arm and left leg.

\section{Case 3}

A 39-year-old male with schizoaffective disorder, polysubstance abuse (K2 and cocaine) presented to the emergency after being found down for an unknown amount of time. At that time, the patient was noted to have swollen and tense right forearm volar and dorsal compartments, with increased blistering, though was noted to be neurovascularly intact distally. He was noted to have tense gluteal and thigh compartments with diminished motor function and sensation in the superficial and deep peroneal nerve distribution. Patient was somnolent but arousable, and was able to follow commands. Vital signs were notable for tachycardia to 118 and labs were notable for an elevated creatinine and a creatine phosphokinase greater than 100,000. The patient underwent emergent fasciotomies of the right deltoid, forearm, wrist, hand, gluteal and thigh compartments. After undergoing multiple irrigation and debridements, the patient required split thickness skin grafts to his right forearm and lateral thigh, which were taken from bilateral anterior thigh donor sites. Upon discharge, the patient's grafts demonstrated 75\% take for the right thigh and $100 \%$ take for the right forearm. At most recent follow-up 12 months postoperatively, the patient was noted to have a rigid contracture of his right middle finger with flexion at the metacarpophalangeal and proximal interphalangeal joints, with an extension of the distal interphalangeal joint, for which the patient underwent a flexor digitorum superficialis tenotomy. Otherwise, the patient had resumed all previous activities.

\section{Case 4}

A 54-year-old male with a history of HIV, seizures, and polysubstance abuse presented to the emergency department with right arm and leg pain and weakness after being found intoxicated in his apartment building. On presentation, the patient was noted to have swollen and tense right forearm volar and dorsal compartments, as well as a firm gluteal compartment. He had pain with passive stretch of both the upper and lower extremity, though was noted to be neurovascularly intact. Vital signs were within normal limits, but labs showed an elevated creatinine, creatine phosphokinase of greater than 100,000, as well as a toxicology screen positive for cocaine and opiates. The patient underwent emergent fasciotomies of both the right forearm volar and dorsal compartments, as well as the hand compartments and the gluteal compartment. After multiple irrigation and debridements to evaluate for necrosing tissue, the patient required a skin graft from his left thigh to his right forearm. After being discharged from the hospital, the patient required admission to a rehabilitation center, though was eventually discharged and was able to resume all daily activities.

\section{Discussion}

\section{Background}

Acute compartment syndrome most commonly affects young males under the age of 35 , likely due to their elevated muscle mass and propensity for higher energy trauma. ${ }^{8}$ Notably, young men are also the most common demographic to suffer from substance abuse and the abuse of opioids, alcohol and prescription drugs has more than doubled in this population in the past decade. ${ }^{9-11}$ All except one of our patients were between the ages of 31 and 40, and all had known history of polysubstance abuse. With the prevalence of substance use disorders on the rise, it is especially critical to be able to diagnose potentially fatal consequences of these substances. Our case series has expanded upon both the presentation of compartment syndrome described in the literature as well as the potential substances that can cause the obtundation and immobility leading to compartment syndrome. In our case series, early recognition of the pathologic process led to improved patient functional outcomes.

\section{Etiology}

Both upper and lower extremity compartment syndromes often present after trauma and are associated with fractures. While compartment syndrome can occur in any fascial compartment, the most common locations are the anterior and lateral compartments of the lower leg. ${ }^{1}$ In a systematic review, upper extremity compartment syndrome was found to be related to fractures in $35 \%$ of the cases, narcotic overdoses in $10 \%$ of the cases and intravenous infiltrations in $8 \%$ of the cases. ${ }^{12,13}$ Gluteal compartment syndrome found to be caused by prolonged 
immobilization is associated with substance abuse in $50 \%$ of the cases, epidural anesthesia in $21 \%$ of the cases, trauma in $21 \%$ of the cases and infection in $7 \%$ of the cases. ${ }^{14}$ While both the upper extremity and gluteal compartments have different etiologies, providers must consider the possibility of having multiple compartment syndromes concurrently.

Depressants, including alcohol and heroin, have been described in the literature in multiple case reports as direct causes of obtundation and immobility leading to compartment syndrome. $^{3-7}$ Other substances such as synthetic cannabinoids or K2 have been less frequently described as being associated with compartment syndrome. In one study from Turkey, Ergul et al describes six cases in which synthetic cannabinoids were directly linked to cases of renal failure, rhabdomyolysis and compartment syndrome. ${ }^{15}$ The authors conclude that due to the increased potency of the newer formulations compared with 9-tetrahydrocannabinol (THC), these drugs have been associated with higher incidences of obtundation and immobility. These reports were analogous to the patients in our case series, as all had signs of rhabdomyolysis as a result of their prolonged immobility. Our case series revealed additional substances that to the authors' knowledge have never been reported in the literature such as benzodiazepines and amphetamines. Therefore, as these drugs become more accessible, healthcare providers should be aware of the substances potential to cause compartment syndrome.

\section{Diagnosis}

The diagnosis of acute compartment syndrome is a clinical one, and any patient in whom a clinician is suspicious of compartment syndrome should undergo emergent fasciotomies as this may lead to improved outcomes. Classically, the five Ps have been associated with compartment syndrome: pain out of proportion, paresthesias, pallor, paralysis and pulselessness. However, these symptoms are often more descriptive of vascular compromise; which can be a component of compartment syndrome. Physical exam often reveals full compartments that are not compressible, pain out of proportion with passive stretch, tenderness to palpation, edema, and erythema surrounding the affected tissue. It is important to note that individual patients may have differing presentations of an evolving compartment syndrome and that by the time an extremity loses pulses, tissue necrosis has likely already begun.
In addition to the physical exam, clinicians can also measure compartment pressures using an intracompartmental pressure monitor. Normal compartment pressures are under $10-15 \mathrm{mmHg} .{ }^{16,17}$ Compartment pressures over $30 \mathrm{~mm} \mathrm{Hg}$ or a difference between diastolic pressure and compartment pressure of less than $30 \mathrm{~mm} \mathrm{Hg}$ leads to critical ischemia of the tissues. ${ }^{16-18}$

Diagnosis of compartment syndrome is especially difficult in patients that are intoxicated or somnolent as they are often unable to fully participate in their exam or articulate their symptoms. Additionally, it can be especially difficult to obtain a history or even know how long and in what position the patient was immobilized. Therefore, in patients presenting in this state, healthcare providers should make sure to have a high clinical suspicion and evaluate the patient appropriately. In all cases reported in this series, no compartment pressures were measured as clinical suspicion was high, and patients were taken emergently to the operating room for fasciotomy. A patient's clinical status can change in a matter of minutes, as seen with the second patient in our case study, and therefore it is especially critical to have a high clinical suspicion to prevent irreversible changes. Even if a patient's presentation is not suggestive of compartment syndrome initially, serial exams are of the utmost importance.

\section{Treatment}

The treatment for a suspected compartment syndrome is emergent fasciotomy and compartment release. Optimal prognosis is associated with performing a fasciotomy within 6 hours of presentation. ${ }^{4}$ A fasciotomy decreases the intra-compartmental pressure, allowing for proper perfusion of the patient's tissues. It is important to release the fascia along the entirety of the compartment to prevent incomplete release and continued tissue damage. After 12 hours, the outcomes associated with performing a fasciotomy are significantly worse. ${ }^{19-21}$ At the time of operation, any non-viable muscle that is encountered should be debrided. A recent study assessed the surgeon's ability to determine muscle viability by comparing biopsies with the surgeon's impression. This study found that in $72 \%$ of the specimens, the surgeon's impression and the histologic appearance differed. ${ }^{22}$ Considering the lack of certainty when assessing muscle viability especially in the acute setting, it is recommended to reexamine at a planned second look within 24-48 hours from the initial 
operation. $^{23}$ This result was echoed in our patients, as all patients required multiple surgical debridements.

Of note, the first and second patients in our study did not require skin grafting. These patients either achieved delayed wound closure or dermal substitutes were applied and secondary closure with repeated dressing changes precluded the need for a skin graft. Since the conclusion of the study, all isolated lower extremity and upper extremity compartment syndrome at this institution have been successfully treated without requiring skin grafting. Given that these patients are often in metabolic disarray on presentation, the ability to forgo skin grafting for the use of dermal substitutes may be beneficial, though this remains to be seen in a clinical trial.

Early diagnosis and appropriate treatment are the key to ensuring good long-term functional outcomes. However, when not managed in the acute setting, patients with compartment syndrome requiring fasciotomy have mortality rates that reach approximately $15 \%{ }^{24}$ When the diagnosis is delayed or missed compartment syndrome can result in sensory deficits, paralysis, muscle contracture, infection, fracture non-union or rhabdomyolysis. ${ }^{1}$

\section{Conclusion}

A patient that presents with symptoms of drug overdose along with painful neurologic deficit should be assessed for compartment syndrome in both upper and lower extremities. As substance use disorder becomes more prevalent and newer drugs such as K2 (synthetic marijuana) become more popular, it is increasingly important for healthcare providers to consider compartment syndrome when a patient presents after protracted immobility in an obtunded state. Early diagnosis is especially critical to prevent the more likely fatal complications.

\section{Ethics Statement}

This research study was authorized by the Columbia University Medical Center IRB, and has been performed in accordance with the principles stated in the Declaration of Helsinki.

\section{Informed Consent Statement}

Written informed consent was obtained from patients to publish case details and accompanying images.

\section{Acknowledgments}

No support was received for this manuscript. Investigation performed at Columbia University Medical Center, New York, New York, USA.

\section{Disclosure}

The authors report no conflicts of interest in this work.

\section{References}

1. Von Keudell AG, Weaver MJ, Appleton PT, et al. Diagnosis and treatment of acute extremity compartment syndrome. Lancet. 2015;386(10000):1299-1310. doi:10.1016/S0140-6736(15)00277-9

2. Friedman ER, Butler KH. Not just another "found down": concomitant upper arm and gluteal compartment syndrome. J Emerg Med. 2018;55(6):e137-e139. doi:10.1016/j.jemermed.2018.09.002

3. Parzych L, Jo J, Diwan A, Swart E. "Found down" compartment syndrome: experience from the front lines of the opioid epidemic. J Bone Joint Surg Am. 2019;101(17):1569-1574. doi:10.2106/ JBJS.18.01307

4. Elkbuli A, Sanchez C, Hai S, et al. Gluteal compartment syndrome following alcohol intoxication: case report and literature review. Ann Med Surg (Lond). 2019;44:98-101. doi:10.1016/j.amsu.2019.07.010

5. Iizuka S, Miura N, Fukushima T, et al. Gluteal compartment syndrome due to prolonged immobilization after alcohol intoxication: a case report. Tokai J Exp Clin Med. 2011;36(2):25-28.

6. Mustafa NM, Hyun A, Kumar JS, et al. Gluteal compartment syndrome: a case report. Cases J. 2009;2(1):190. doi:10.1186/17571626-2-190

7. Sofat N, Bell S, Turner J, et al. A case of acute renal failure and compartment syndrome after an alcoholic binge. J Accid Emerg Med. 1999;16(4):296-298. doi:10.1136/emj.16.4.296

8. Torlincasi AM, Lopez RA, Waseem M. Acute Compartment Syndrome. StatPearls [Internet]. Treasure Island (FL): StatPearls Publishing; 2020:24.

9. Substance Abuse and Mental Health Services Administration. Key Substance Use and Mental Health Indicators in the United States: Results from the 2017 National Survey on Drug Use and Health (HHS Publication No. SMA 18-5068, NSDUH Series H-53). Rockville, MD: Center for Behavioral Health Statistics and Quality, Substance Abuse and Mental Health Services Administration; 2018. Available from: https://www.samhsa.gov/data. Accessed December 24, 2020.

10. Centers for Disease Control and Prevention. Today's Heroin Epidemic. 2015. Available from: https://www.cdc.gov/vitalsigns/her oin/index.html. Accessed December 24, 2020.

11. Substance Abuse and Mental Health Services Administration. Results from the 2017 National Survey on Drug Use and Health: Detailed Tables. 2018. Available from: https://www.samhsa.gov/data/sites/ default/files/cbhsq-reports/NSDUHDetailedTabs2017/ NSDUHDetailedTabs2017.pdf. Accessed December 24, 2020.

12. Roberts CS, Gorczyca JT, Ring D, et al. Diagnosis and treatment of less common compartment syndromes of the upper and lower extremities: current evidence and best practices. Instr Course Lect. 2011;60:43-50.

13. Elliott KGB, Johnstone AJ. Diagnosing acute compartment syndrome. $J$ Bone Joint Surg Br. 2003;85-B(5):625-632. doi:10.1302/0301-620X.85B5.14352

14. Henson JT, Roberts CS, Giannoudis PV. Gluteal compartment syndrome. Acta Orthop Belg. 2009;75(2):147-152.

15. Ergul DF, Ekemen S, Yelken BB. Synthetic cannabinoid 'bonzai' intoxication: six case series. Turk J Anaesthesiol Reanim. 2015;43 (5):347-351. doi:10.5152/TJAR.2015.05668

16. Malinoski DJ, Slater MS, Mullins RJ. Crush injury and rhabdomyolysis. Crit Care Clin. 2004;20(1):171-192. doi:10.1016/ S0749-0704(03)00091-5

17. Perron AD, Brady WJ, Keats TE. Orthopedic pitfalls in the ED: acute compartment syndrome. Am J Emerg Med. 2001;19(5):413-416. doi:10.1053/ajem.2001.24464 
18. Smith J, Greaves I. Crush injury and crush syndrome: a review. $J$ Trauma. 2003;54(5 Suppl):S226-S230. doi:10.1097/01. TA.0000047203.00084.94

19. Lachiewicz PF, Latimer HA. Rhabdomyolysis following total hip arthroplasty. $J$ Bone Joint Surg Br. 1991;73(4):576-579. doi:10.1302/0301-620X.73B4.2071638

20. Gaines RJ, Randall CJ, Browne KL, et al. Delayed presentation of compartment syndrome of the proximal lower extremity after low-energy trauma in patients taking warfarin. Am J Orthop (Belle Mead NJ). 2008;37(12):E201-E204.

21. Chochola M, Lubanda JC, Skalicka L, et al. Bilateral leg compartment syndrome due to severe myonecrosis caused by inappropriate use of simvastatin. J Mal Vasc. 2008;33(4-5):229-233. doi:10.1016/j. jmv.2008.07.092
22. Sasoon A, Riehl J, Rich A, et al. Muscle viability revisited: are we removing normal muscle? A critical evaluation of dogmatic debridement. J Orthop Trauma. 2016;30(1):17-21. doi:10.1097/ BOT.0000000000000423

23. Cone J, Inaba K. Lower extremity compartment syndrome. Trauma Surg Acute Care Open. 2017;2:1-6. doi:10.1136/tsaco-2017-000094

24. Heemskerk J, Kitslaar P. Acute compartment syndrome of the lower leg: retrospective study on prevalence, technique, and outcome of fasciotomies. World J Surg. 2003;27(6):744-747. doi:10.1007/ s00268-003-6691-7

\section{Publish your work in this journal}

Orthopedic Research and Reviews is an international, peer-reviewed, open access journal that focusing on the patho-physiology of the musculoskeletal system, trauma, surgery and other corrective interventions to restore mobility and function. Advances in new technologies, materials, techniques and pharmacological agents are particularly welcome. The manuscript management system is completely online and includes a very quick and fair peer-review system, which is all easy to use. Visit http://www.dovepress.com/testimonials.php to read real quotes from published authors. 